\title{
New parametrization of the seesaw mechanism and applications in supersymmetric models
}

\author{
John Ellis, ${ }^{1}$ Junji Hisano, ${ }^{2}$ Martti Raidal, ${ }^{1,3}$ and Yasuhiro Shimizu ${ }^{4}$ \\ ${ }^{1}$ CERN, CH 1211 Geneva 23, Switzerland \\ ${ }^{2}$ ICRR, University of Tokyo, Kashiwa 277-8582, Japan \\ ${ }^{3}$ National Institute of Chemical Physics and Biophysics, Tallinn 10143, Estonia \\ ${ }^{4}$ Department of Physics, Nagoya University, Nagoya 464-8692, Japan
}

(Received 19 June 2002; published 31 December 2002)

\begin{abstract}
We present a new parametrization of the minimal seesaw model, expressing the heavy-singlet neutrino Dirac Yukawa couplings $\left(Y_{\nu}\right)_{i j}$ and Majorana masses $M_{N_{i}}$ in terms of effective light-neutrino observables and an auxiliary Hermitian matrix $H$. In the minimal supersymmetric version of the seesaw model, the latter can be related directly to other low-energy observables, including processes that violate charged lepton flavor and $C P$. This parametrization enables one to respect the stringent constraints on muon-number violation while studying the possible ranges for other observables by scanning over the allowed parameter space of the model. Conversely, if any of the lepton-flavor-violating process is observed, this measurement can be used directly to constrain $\left(Y_{\nu}\right)_{i j}$ and $M_{N_{i}}$. As applications, we study flavor-violating $\tau$ decays and the electric dipole moments of leptons in the minimal supersymmetric seesaw model.
\end{abstract}

DOI: $10.1103 /$ PhysRevD.66.115013

PACS number(s): 11.30.Pb, 12.60.Jv

\section{INTRODUCTION}

Experiments on both atmospheric and solar neutrinos have now provided "smoking guns" for neutrino oscillations. Most recently, the direct SNO measurement of the solar neutrino flux via neutral-current scattering confirms solar neutrino oscillations [1] and favors strongly the large mixing angle (LMA) solution [2]. This region of parameter space is within reach of the KamLAND experiment, and is expected to be probed soon [3]. The existence of large mixing angles for both solar and atmospheric neutrinos [4] is one of the biggest mysteries in particle physics. The most favored mechanism for generating neutrino masses is the seesaw mechanism [5], which naturally explains their small sizes. However, it is an open question whether the seesaw mechanism can explain why mixing in the lepton sector seems to be larger than in the quark sector. In the absence of a theory of flavor, it is important to study the consequences of neutrino mixing for as many physical observables as possible.

In the minimal supersymmetric seesaw model, leptonflavor-violating (LFV) phenomena provide a tool to study indirectly neutrino parameters and probe other aspects beyond the large mixing angles measured in neutrino oscillations. If supersymmetry breaking originates from physics beyond the heavy singlet neutrino mass scale, LFV slepton masses are induced radiatively [6,7] via the Dirac Yukawa couplings of the neutrinos, even if the input supersymmetrybreaking parameters are flavor blind. On the other hand, the light neutrino masses and mixings depend on both the Yukawa couplings and the Majorana masses of the heavy singlet neutrinos. Thus one can hope to reconstruct the physical parameters in the heavy singlet-neutrino sector entirely in terms of the light neutrino data and low-energy observables such as rates for LFV processes [8]. To this end, in this paper we present a parametrization of the minimal seesaw model and apply it to the minimal supersymmetric version of the seesaw model.

The essence of our parametrization is the following. The minimal seesaw mechanism, whether supersymmetric or non-supersymmetric, involves 18 physical degrees of freedom, including 6 real mixing angles and $6 C P$-violating phases. On the other hand, the induced light-neutrino mass matrix has 9 degrees of freedom, including 3 real mixing angles and $3 C P$-violating phases. Thus we need 9 additional degrees of freedom to parametrize completely the seesaw mechanism. These can be chosen in such a way as to be related to low-energy leptonic observables in the supersymmetric version of the seesaw model. We recall that the LFV renormalization of the supersymmetry-breaking parameters at low energy are proportional to

$$
H_{i j}=\sum_{k}\left(Y_{\nu}^{*}\right)_{k i}\left(Y_{\nu}\right)_{k j} \log \frac{M_{G}}{M_{N_{k}}},
$$

where $\left(Y_{\nu}\right)$ and $M_{N}$ are the heavy singlet-neutrino Dirac Yukawa couplings and Majorana masses, respectively, and $M_{G}$ is the grand unified theory (GUT) scale where the initial conditions for the supersymmetry-breaking parameters are imposed. Since $H$ is a Hermitian matrix, it has 9 degrees of freedom including 3 real mixings and 3 phases. This implies that we can parametrize the seesaw mechanism by the light neutrino mass matrix $\mathcal{M}_{\nu}$ and the Hermitian matrix $H$ according to

$$
\left(\mathcal{M}_{\nu}, H\right) \rightarrow\left(Y_{\nu}, M_{N}\right) .
$$

As a result, we can obtain $Y_{\nu}$ and $M_{N}$ that yield automatically the light neutrino masses and mixings measured in oscillation experiments. However, the main motivation for our parametrization comes from its power in studies of the charged-lepton physics in the supersymmetric seesaw model. ${ }^{1}$

\footnotetext{
${ }^{1}$ We emphasize, though, that the parametrization itself is more general, and does not depend on the existence of supersymmetry.
} 
The LMA solution to the solar neutrino anomaly tends to predict a large branching ratio for $\mu \rightarrow e \gamma$ in the supersymmetric seesaw model [9-11], which may be within reach of near-future experiments, or even beyond the current experimental bound. ${ }^{2}$ This does not imply that the supersymmetric seesaw model is strongly constrained, because it has a multidimensional parameter space. However, it is difficult to scan efficiently over the allowed parameter space while satisfying the $\mu \rightarrow e \gamma$ constraint. Our parametrization solves this difficulty, because the parameter matrix $H$ is related to the solutions of the renormalization-group equations. Therefore, it is straightforward to choose a parameter region where $\mu \rightarrow e \gamma$ is suppressed, but the other low-energy observables may vary over their full ranges. Furthermore, if some future experiment discovers a LFV process or the electric dipole moment (EDM) of some lepton, this observation will be directly related to $H$ and thus to the neutrino parameters. In our parametrization, the high-energy neutrino couplings and masses can be expressed entirely in terms of the induced low-energy observables.

Our work is organized as follows. In Sec. II we outline the new parametrization and our procedure for analyzing charged-lepton decays. In Sec. III we explain the relation between our parametrization and the physical observables. In Sec. IV we present a study of LFV $\tau$ decays and the EDMs of the electron and muon in the supersymmetric seesaw model, as applications of our approach. We find that $\tau$ $\rightarrow \mu(e) \gamma$ can saturate the current experimental bound, even when $\mu \rightarrow e \gamma$ is suppressed enough to be acceptable. The EDMs of the muon and electron generally fall below $10^{-27}\left(10^{-29}\right) e \mathrm{~cm}$ in our random parameter scan. We also present the relation between $\operatorname{Br}(\tau \rightarrow \mu(e) \gamma)$ and $\operatorname{Br}(\tau$ $\left.\rightarrow \mu(e) \ell^{+} \ell^{-}\right)$. Section V summarizes our conclusions.

\section{PARAMETRIZATION OF NEUTRINO COUPLINGS AND MASSES}

In view of the subsequent application to the supersymmetric version of the seesaw model, we illustrate the parametrization for this case, though it is also valid in the absence of supersymmetry. The leptonic superpotential of the supersymmetric version of the minimal seesaw model is

$$
W=N_{i}^{c}\left(Y_{\nu}\right)_{i j} L_{j} H_{2}+E_{i}^{c}\left(Y_{e}\right)_{i j} L_{j} H_{1}+\frac{1}{2} N^{c}{ }_{i}\left(M_{N}\right)_{i j} N_{j}^{c},
$$

where the indices $i, j$ run over three generations and $\left(M_{N}\right)_{i j}$ is the heavy singlet-neutrino mass matrix. In addition to the three charged-lepton masses, this superpotential has 18 physical parameters, including 6 real mixing angles and 6 $C P$-violating phases.

At low energies, the effective superpotential obtained by integrating out the heavy neutrinos is

\footnotetext{
${ }^{2}$ Also, some explicit models predict the third neutrino mixing parameter $U_{e 3}$ to be $\mathcal{O}\left(10^{-(1-2)}\right)$, which may also lead to a large branching ratio for $\mu \rightarrow e \gamma[12]$.
}

$$
W_{\mathrm{eff}}=E_{i}^{c}\left(Y_{e}\right)_{i} L_{j} H_{1}+\frac{1}{2 v^{2} \sin ^{2} \beta}\left(\mathcal{M}_{\nu}\right)_{i j}\left(L_{i} H_{2}\right)\left(L_{j} H_{2}\right),
$$

where we work in a basis in which the charged-lepton Yukawa couplings are diagonal. The second term in Eq. (4) leads to the light neutrino masses and mixings. The explicit form of $\mathcal{M}_{\nu}$ is given by

$$
\left(\mathcal{M}_{\nu}\right)_{i j}=\sum_{k} \frac{\left(Y_{\nu}\left(Q_{k}\right)\right)_{k i}\left(Y_{\nu}\left(Q_{k}\right)\right)_{k j}}{M_{N_{k}}} v^{2} \sin ^{2} \beta
$$

where the heavy-singlet neutrino Dirac Yukawa couplings $Y_{\nu}$ and masses $M_{N_{i}}$ are defined at the renormalization scale $Q_{k}$ $=M_{N_{k}}$, and in our notation $M_{N_{1}}<M_{N_{2}}<M_{N_{3}}$. It is important to distinguish between the renormalization scales for different components in the Yukawa coupling matrix, since the EDMs of charged leptons in the supersymmetric seesaw model are sensitive to non-universal radiative corrections to the supersymmetry-breaking parameters, which come from the non-degeneracy of the heavy singlet neutrino masses [13]. For simplicity, we ignore the renormalization of $\mathcal{M}_{\nu}$ after the decoupling of the singlet neutrinos.

The light neutrino mass matrix $\mathcal{M}_{\nu}(5)$ is symmetric, with 9 parameters, including 3 real mixing angles and 3 $C P$-violating phases. It can be diagonalized by a unitary matrix $U$ as

$$
U^{T} \mathcal{M}_{\nu} U=\mathcal{M}_{\nu}^{D} .
$$

By redefinition of fields one can rewrite $U \equiv V P$, where $P$ $\equiv \operatorname{diag}\left(e^{i \phi_{1}}, e^{i \phi_{2}}, 1\right)$ and $V$ is the Maki-Nakagawa-Sakata (MNS) matrix, with the 3 real mixing angles and the remaining $C P$-violating phase.

The key proposal of this paper is to characterize the seesaw neutrino sector by $\mathcal{M}_{\nu}$ and a Hermitian matrix $H$, whose diagonal terms are real and positive, which is defined in terms of $Y_{\nu}$ and the heavy neutrino masses $M_{N}$ by

$$
H_{i j}=\sum_{k}\left(Y_{\nu}^{*}\left(Q_{k}\right)\right)_{k i}\left(Y_{\nu}\left(Q_{k}\right)\right)_{k j} \log \frac{M_{G}}{M_{N_{k}}},
$$

with $M_{G}$ the GUT scale. The Hermitian matrix $H$ has 9 parameters including 3 phases, which are clearly independent of the parameters in $\mathcal{M}_{\nu}$. Thus $\mathcal{M}_{\nu}$ and $H$ together provide the required 18 parameters, including $6 C P$-violating phases.

Although our parametrization also includes an unphysical region, it has the merit of suitability for comprehensive studies of the minimal supersymmetric seesaw model. In this model, the non-universal elements in the left-handed slepton mass matrix, which induce the charged LFV observables, are approximately proportional to $H$ if the slepton masses are flavor independent at $M_{G}$. Thus, this parametrization allows us to control the LFV processes and scan over the allowed parameter space at the same time. Conversely, if some LFV process is discovered in the future, its measurement can be incoprorated directly into our parametrization of the neutrino sector. 
We now explain how to reconstruct the heavy singletneutrino sector from knowledge of $\mathcal{M}_{\nu}$ and $H$. First we recall the parametrization of the neutrino Dirac Yukawa coupling given in [10],

$$
\left(Y_{\nu}\left(Q_{i}\right)\right)_{i j}=\left.\frac{\sqrt{M_{N}} R \sqrt{\mathcal{M}_{\nu}} U^{\dagger}}{v \sin \beta}\right|_{i j},
$$

where $R$ is an auxiliary complex orthogonal matrix: $R R^{T}$ $=R^{T} R=1$. Using this parametrization, $H$ becomes

$$
H=\frac{1}{v^{2} \sin ^{2} \beta} U \sqrt{\mathcal{M}_{\nu}} R^{\dagger} \overline{M_{N}} R \sqrt{\mathcal{M}_{\nu}} U^{\dagger}
$$

where $\overline{M_{N_{i}}} \equiv M_{N_{i}} \log \left(M_{G} / M_{N_{i}}\right)$. If we can diagonalize the following Hermitian matrix $H^{\prime}$,

$$
H^{\prime}={\sqrt{\mathcal{M}_{\nu}}}^{-1} U^{\dagger} H U{\sqrt{\mathcal{M}_{\nu}}}^{-1} v^{2} \sin ^{2} \beta,
$$

by the complex orthogonal matrix $R^{\prime}$ :

$$
H^{\prime}=R^{\prime \dagger} \overline{M_{N}} R^{\prime},
$$

then we can calculate the heavy singlet neutrino masses from $\overline{M_{N}}$ and the corresponding $Y_{\nu}$ from Eq. (8) taking $R=R^{\prime}$.

However, the Hermitian matrix $H^{\prime}$ cannot always be diagonalized by a complex orthogonal matrix: the condition for such a diagonalization is that all the eigenvalues of $H^{\prime \star} H^{\prime}$ are positive, in which case $R^{\prime}$ is given by the eigenvectors of $H^{\prime \star} H^{\prime}$. This reflects the fact that our parametrization also includes an unphysical region, so that every chosen $H$ does not necessarily give physical neutrino masses and couplings. Since our objective in this paper is to survey the multi-dimensional parameter space using scatter plots, this shortcoming is not critical.

In our subsequent analysis, we first generate randomly the matrix $H$, the phases and the common mass scale in the light neutrino sector, and then calculate the corresponding heavy neutrino masses and couplings. The Yukawa couplings $\left(Y_{\nu}\right)_{i j}$ contribute to the renormalization-group (RG) equations above $M_{N_{i}}$, since the corresponding singlet neutrino is dynamical there. When we derive the Yukawa couplings at the GUT scale, we introduce $\left(Y_{\nu}\right)_{i j}$ in the RG equations at $Q_{i}$ $=M_{N_{i}}$ where the neutrinos appear. When evaluating the supersymmetry-breaking parameters at the weak scale, the right-handed neutrinos are integrated out at their own mass scales.

\section{OBSERVABLES}

In the previous section we presented our parametrization of the minimal seesaw mechanism in terms of the lightneutrino mass matrix $\mathcal{M}_{\nu}$ and a Hermitian parameter matrix $H$. Here we make explicit the correspondence between this parametrization and low-energy observables in the supersymmetric version of the seesaw model.

\section{A. Neutrino experiments}

As already mentioned, the light-neutrino mass matrix $\mathcal{M}_{\nu}$ contains nine physical parameters: 3 mass eigenvalues, 3 mixing angles, $1 C P$-violating mixing phase in the MNS matrix, and $2 C P$-violating Majorana phases, the LMA solution is now favored, following the SNO neutral-current result. Thus, the favored regions for the atmospheric and solar neutrino parameters are

$$
\begin{aligned}
\Delta m_{32}^{2} & =(1-5) \times 10^{-3} \mathrm{eV}^{2}, \\
\sin ^{2} 2 \theta_{23} & =(0.8-1.0), \\
\Delta m_{21}^{2} & =10^{-(4-5)} \mathrm{eV}^{2}, \\
\tan ^{2} \theta_{12} & \simeq(0.2-0.6) .
\end{aligned}
$$

The CHOOZ [14] and Palo Verde [15] experiments provide the constraint

$$
\sin ^{2} 2 \theta_{13} \lesssim 0.1 \text {. }
$$

These parameters, together with the $C P$-violating mixing phase in the MNS matrix, may be measured in future experiments, such as the KamLAND and the neutrino factory. There would still be three undetermined parameters, the normalization of the neutrino mass and the Majorana phases. The neutrinoless double beta decay matrix element is proportional to

$$
\left|m_{e e}\right|=\left|\sum_{i} U_{e i}^{*} m_{\nu_{i}} U_{i e}^{\dagger}\right|,
$$

and so would provide a constraint on the neutrino mass scale and Majorana phases, if it could be measured.

\section{B. Charged LFV processes}

If the supersymmetry-breaking parameters at the GUT scale are universal, off-diagonal components in the lefthanded slepton mass matrix $m_{\tilde{L}}$ and the trilinear slepton coupling $A_{e}$ are induced by renormalization, taking the approximate forms

$$
\begin{aligned}
& \left(\delta m_{\tilde{L}}^{2}\right)_{i j} \simeq-\frac{1}{8 \pi^{2}}\left(3 m_{0}^{2}+A_{0}^{2}\right) H_{i j}, \\
& \left(\delta A_{e}\right)_{i j} \simeq-\frac{1}{8 \pi^{2}} A_{0} Y_{e_{i}} H_{i j},
\end{aligned}
$$

where $i \neq j$, and the off-diagonal components of the righthanded slepton mass matrix are suppressed. The parameters $m_{0}$ and $A_{0}$ are the universal scalar mass and trilinear coupling at the GUT scale. Here, we ignore terms of higher order in $Y_{e}$, assuming that $\tan \beta$ is not extremely large. Thus, the parameters in $H$ may in principle be determined by the LFV processes of charged leptons. Currently, $\mu \rightarrow e \gamma$ experiments give the following constraints on them: 


$$
\begin{gathered}
H_{12} \lesssim 10^{-2} \times \tan ^{-1} \beta\left(\frac{m_{0}}{100 \mathrm{GeV}}\right)^{2}\left(\frac{\operatorname{Br}(\mu \rightarrow e \gamma)}{1.2 \times 10^{-11}}\right)^{1 / 2}, \\
H_{13} H_{32} \lesssim 10^{-1} \times \tan ^{-1} \beta\left(\frac{m_{0}}{100 \mathrm{GeV}}\right)^{2}\left(\frac{\operatorname{Br}(\mu \rightarrow e \gamma)}{1.2 \times 10^{-11}}\right)^{1 / 2},
\end{gathered}
$$

where we take $\left(m_{\tilde{L}}^{2}\right)_{i i} \sim m_{0}^{2}$. These components may also be measured directly in future collider experiments, if the sleptons are produced there $[16,17]$.

Although the matrix $H$ has three $C P$-violating phases, two of them are almost irrelevant to charged LFV phenomena. The two phases may be moved from $H$ to $\mathcal{M}_{\nu}$ by a rotation of $L$. In fact, there is only a single Jarlskog invariant obtainable from $H[16]$ :

$$
J=\operatorname{Im} H_{12} H_{23} H_{31},
$$

which determines the $T$-odd asymmetry in $\mu \rightarrow 3 e$ [18].

We kept in Eq. (18) only the leading-order contributions to the soft supersymmetry-breaking parameters, and ignored higher-order corrections. If some components of $H$ are suppressed, non-trivial flavor structure may emerge in the higher-order corrections. At $\mathcal{O}\left(\log ^{2} M_{G} / M_{N_{3}}\right)$ or $\mathcal{O}\left(\log M_{G} / M_{N_{3}} \log M_{N_{j}} / M_{N_{i}}\right)(i \neq j),\left(m_{\tilde{L}}^{2}\right)$ and $\left(A_{e}\right)$ get the following corrections:

$$
\begin{aligned}
\left(\delta^{\prime} m_{\tilde{L}}^{2}\right)_{i j} \simeq & \frac{1}{(4 \pi)^{4}}\left(A_{0}^{2} H^{2}\right)_{i j}+\frac{6}{(4 \pi)^{4}} \\
& \times\left(3 m_{0}-A_{0}^{2}\right) \sum_{k<l}\left(\left\{X_{k}, X_{l}\right\} \log \frac{M_{N_{l}}}{M_{N_{k}}}\right) \log \frac{M_{G}}{M_{N_{3}}}, \\
\left(\delta^{\prime} A_{e}\right)_{i j} \simeq & \frac{1}{(4 \pi)^{4}} A_{0} Y_{e_{i}} \sum_{k<l}\left[6\left\{X_{k}, X_{l}\right\} \log \frac{M_{N_{l}}}{M_{N_{k}}}\right. \\
& \left.+4\left[X_{k}, X_{l}\right] \log \frac{M_{N_{l}}}{M_{N_{k}}}\right]_{i j} \log \frac{M_{G}}{M_{N_{3}}},
\end{aligned}
$$

where

$$
\left(X_{k}\right)_{i j}=\left(Y_{\nu}^{*}\left(M_{N_{k}}\right)\right)_{k i}\left(Y_{\nu}\left(M_{N_{k}}\right)\right)_{k j} .
$$

The second term in Eq. (21) and the term in Eq. (22) come from threshold corrections at the heavy singlet-neutrino scale, due to the non-degeneracy of their neutrino masses.

\section{EDMs of the charged leptons}

The EDMs of the charged leptons depend non-trivially on the parameters in the supersymmetric seesaw model. If the heavy singlet-neutrino masses are degenerate, the EDMs of the charged leptons are strongly suppressed by the chiral structure of the seesaw model, and are proportional to

$$
\operatorname{Im}\left[\left[Y_{e} Y_{\nu}^{\dagger} Y_{\nu}\left[Y_{e}^{\dagger} Y_{e}, Y_{\nu}^{\dagger} Y_{\nu}\right] Y_{\nu}^{\dagger} Y_{\nu}\right]_{i i}\right]
$$

This is similar to the neutron EDM in the standard model. However, when the heavy singlet-neutrino masses are not degenerate, the EDMs may be enhanced significantly. The trilinear coupling $A_{e}$ gets a threshold correction at the heavy singlet neutrino scale, and may get radiatively-induced diagonal phases proportional to

$$
\operatorname{Im}\left[X_{j}, X_{k}\right]_{i i} \log M_{N_{k}} / M_{N_{j}} \neq 0,
$$

as in Eq. (22) [13]. This depends non-trivially on the $C P$-violating phases, including the two Majorana phases in $\mathcal{M}_{\nu}$ and two phases in $H$ that are irrelevant for LFV.

\section{PHENOMENOLOGICAL ANALYSIS}

Using the parametrization proposed above, we now study the branching ratios for LFV $\tau$ decays, such as $\operatorname{Br}(\tau$ $\rightarrow \mu \gamma), \operatorname{Br}(\tau \rightarrow e \gamma)$ and $\operatorname{Br}(\tau \rightarrow 3 l)$, and the EDMs $d_{\mu}$ and $d_{e}$. We fix the light neutrino parameters: $\Delta m_{32}^{2}=3$ $\times 10^{-3} \mathrm{eV}^{2}, \quad \Delta m_{21}^{2}=4.5 \times 10^{-5} \mathrm{eV}^{2}, \quad \tan ^{2} \theta_{23}=1 \quad$ and $\tan ^{2} \theta_{12}=0.4$ corresponding to the LMA solution for the solar neutrino anomaly. Since the bound on the angle $\theta_{13}$ is quite stringent, leptonic observables are dominated by the other free parameters and our results depend very weakly on its actual value. We fix $\sin \theta_{13}=0.1$ and $\delta=\pi / 2$. Plots for $\sin \theta_{13}=0$ are indistinguishable from the presented ones. We study both the normal and the inverse hierarchy of light neutrino masses, since neutrino oscillations do not discriminate between these two cases. ${ }^{3}$ As input parameters, we then have the lightest effective neutrino mass $m_{1}$ (or $m_{3}$ for inversely ordered neutrinos), which we generate in the range $\left(10^{-4}\right.$ $-0.3) \mathrm{eV}$, the two low scale Majorana phases $\phi_{1,2}$ and the matrix $H$, which we generate randomly.

We study two different limits of the parameter matrix $H$, of the form

$$
H_{1}=\left(\begin{array}{ccc}
a & 0 & 0 \\
0 & b & d \\
0 & d^{\dagger} & c
\end{array}\right),
$$

and

$$
H_{2}=\left(\begin{array}{ccc}
a & 0 & d \\
0 & b & 0 \\
d^{\dagger} & 0 & c
\end{array}\right),
$$

where $a, b, c$ are real and positive, and $d$ is a complex number. We sample these parameters randomly in the range $10^{-2}<a, b, c,|d|<10$, with distributions that are flat on a logarithmic scale. Also, we require the Yukawa couplingsquared to be smaller than $4 \pi$, so that $Y_{\nu}$ remains perturbative up to $M_{G}$.

\footnotetext{
${ }^{3}$ Future neutrinoless double-beta decay and oscillation experiments will resolve this ambiguity.
} 

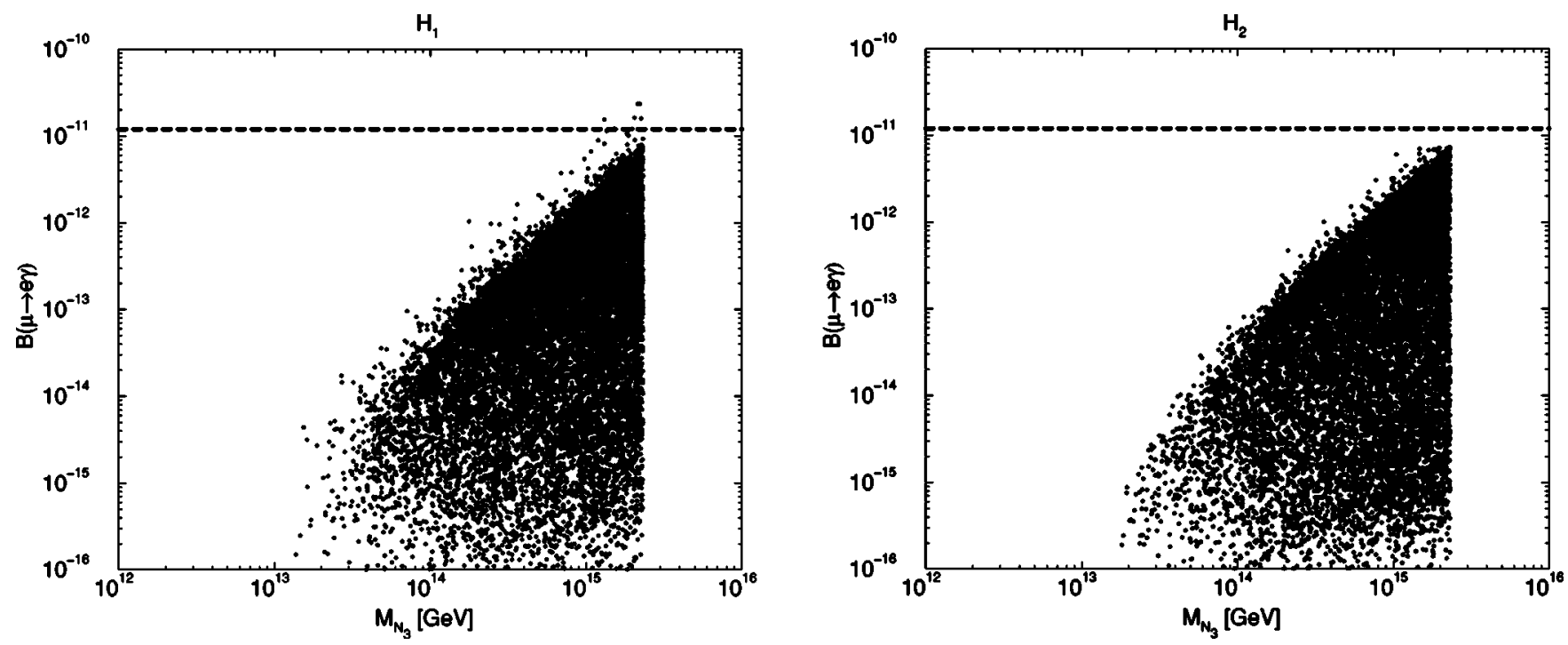

FIG. 1. Scatter plot of $\operatorname{Br}(\mu \rightarrow e \gamma)$ against the heaviest singlet neutrino mass $M_{N_{3}}$ for the ansatz (a) $H_{1}$ and (b) $H_{2}$. We take the SU(2) gaugino mass to be $200 \mathrm{GeV}, m_{0}=100 \mathrm{GeV}, A_{0}=0 \mathrm{GeV}, \tan \beta=10$ and $\operatorname{sgn}(\mu)=+1$. Other input parameters are specified in the text.

In the above ansatz, we take $H_{12}=0$ and $H_{13} H_{32}=0$ because these conditions suppress $\operatorname{Br}(\mu \rightarrow e \gamma)$, as seen in Eq. (19). We show $\operatorname{Br}(\mu \rightarrow e \gamma)$ as a function of the heaviest right-handed neutrino mass $M_{N_{3}}$ for the two structures $H_{1}$ and $H_{2}$ in Fig. 1. As a reference, here we take the $\mathrm{SU}(2)$ gaugino mass to be $200 \mathrm{GeV}, m_{0}=100 \mathrm{GeV}, A_{0}=0 \mathrm{GeV}$, $\tan \beta=10$ and $\operatorname{sgn}(\mu)=+1$.

With the chosen forms $H_{1}$ and $H_{2}, \operatorname{Br}(\mu \rightarrow e \gamma)$ is suppressed in a broad range of parameters. In these figures $\mu$ $\rightarrow e \gamma$ is induced entirely by $\mathcal{O}\left(\log M_{G} / M_{N_{3}} \log M_{N_{j}} / M_{N_{i}}\right)(i$ $\neq j$ ) corrections to the slepton mass matrix and the slepton trilinear coupling (21), (22). Thus, if all non-zero components in $H_{1}$ or $H_{2}$ are of order unity, the correction to $\left(m_{\tilde{L}}^{2}\right)_{12}$ is not necessary negligible, and one may find values of the branching ratio above the present experimental bound. We also see in Fig. 1 that improving the present sensitivity to $\operatorname{Br}(\mu \rightarrow e \gamma)$ by three orders of magnitude, which is currently being undertaken at PSI [19], would be interesting for a large fraction of the models studied. The MECO and PRISM searches for $\mu^{-}-e^{-}$conversion on nuclei [20] would also be interesting in this respect, and the sensitivities to both processes could be improved at the front end of a neutrino factory [21].

The ansatz $H_{1}$ minimizes $\tau \longrightarrow e \gamma$ while $\tau \rightarrow \mu \gamma$ can be large, and the opposite is the case for $\mathrm{H}_{2}$, since these processes are sensitive to $H_{13}$ and $H_{23}$, respectively. Similarly, the EDM of the muon can be maximized in $H_{1}$ while the electron EDM can be large in $H_{2}$. We exhibit these results in the following subsections.

\section{A. LFV $\tau$ decay}

In Fig. 2 we present $\operatorname{Br}(\tau \rightarrow \mu \gamma)$ for the ansatz $H_{1}$, assuming either the normal or the inverted hierarchy for the light neutrino mass spectrum. The horizontal axis is the lightest stau mass $m_{\tau_{1}}$, and the other supersymmetry-breaking parameters are determined by choosing the SU(2) gaugino mass to be $200 \mathrm{GeV}, A_{0}=0, \mu>0$, and $\tan \beta=10$ and 30 . The parameters in $\mathcal{M}_{\nu}$ and $H$ are the same as in Fig. 1. The branching ratio scales as $\tan ^{2} \beta$. We see from these figures that the branching ratio is similar for the normal and inverted hierarchies of light-neutrino masses. In our parametrization the branching ratio is determined mainly by $H$ and the sparticle mass spectrum. The dependence on the details of $\mathcal{M}_{\nu}$ appears through the heavy singlet neutrino masses, which influence the branching ratios only logarithmically. We find that $\operatorname{Br}(\tau \rightarrow \mu \gamma)$ can reach even above the present experimental bound, attaining $10^{-4}\left(10^{-5}\right)$ for $\tan \beta=30(10)$, these limits are arising from the perturbative bound on the neutrino Yukawa coupling.

Next we show $\operatorname{Br}(\tau \rightarrow e \gamma)$ for the ansatz $\mathrm{H}_{2}$ in Fig. 3. The input parameters are the same as in Fig. 2, and the behavior is similar to that of $\operatorname{Br}(\tau \rightarrow \mu \gamma)$ in Fig. 2. This process may also reach to $10^{-4}\left(10^{-5}\right)$ for $\tan \beta=30(10)$.

We see in Figs. 2, 3 that improving the present sensitivity to $\operatorname{Br}(\tau \rightarrow \mu / e \gamma)$ by two orders of magnitude, which seems feasible with the present generation of $B$ factories [22], would be very interesting for many of the models studied. Whilst the $B$ factories have good sensitivities to $\tau \rightarrow \mu \gamma$ and $\tau \rightarrow e \gamma$, hadron colliders may have better reach for $\tau \rightarrow 3 \ell$ decays. We present here a simple formula for the LFV $\tau$ decays to three charged leptons. When $\left(m_{\tilde{L}}^{2}\right)_{23}$ is nonvanishing, non-zero $\tau \rightarrow \mu \gamma$ and $\tau \rightarrow \mu \ell^{+} \ell^{-}$are both predicted. The photonic penguin diagram tends to dominate over other contributions in the trilepton final state due to the phase-space integral. When $\tan \beta$ is large, the dominance is even stronger. When the photonic penguin diagram is dominant in $\tau \rightarrow \mu \ell^{+} \ell^{-}$,

$$
\begin{aligned}
& \frac{\operatorname{Br}(\tau \rightarrow 3 \mu)}{\operatorname{Br}(\tau \rightarrow \mu \gamma)}=\frac{\alpha}{8 \pi} \frac{8}{3}\left(\log \frac{m_{\tau}^{2}}{m_{\mu}^{2}}-\frac{11}{4}\right) \simeq \frac{1}{440}, \\
& \frac{\operatorname{Br}(\tau \rightarrow \mu 2 e)}{\operatorname{Br}(\tau \rightarrow \mu \gamma)}=\frac{\alpha}{8 \pi} \frac{8}{3}\left(\log \frac{m_{\tau}^{2}}{m_{e}^{2}}-\frac{8}{3}\right) \simeq \frac{1}{94} .
\end{aligned}
$$



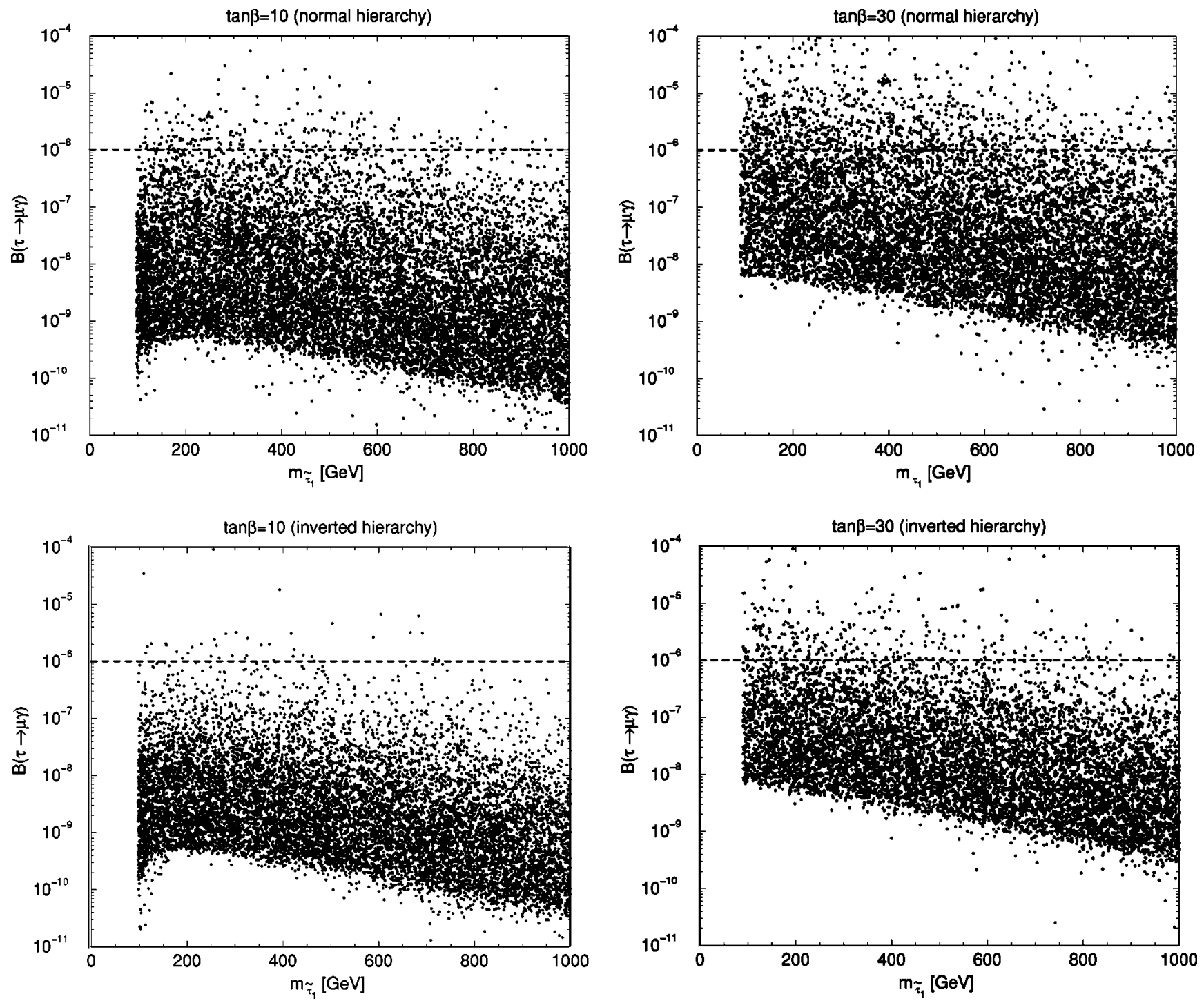

FIG. 2. Scatter plot of $\operatorname{Br}(\tau \rightarrow \mu \gamma)$ against the lightest stau mass for the ansatz $H_{1}$. We take the $\mathrm{SU}(2)$ gaugino mass to be $200 \mathrm{GeV}$, $A_{0}=0, \mu>0$, and $\tan \beta=10$ and 30 . We consider both the normal and inverted hierarchies for the light neutrino mass spectrum.

The branching ratio for $\mu e^{+} e^{-}$is larger than that to $\mu \mu^{+} \mu^{-}$, because the phase space is larger. Similarly, we get the following relations for the $\tau-e$ transition:

$$
\begin{gathered}
\frac{\mathrm{Br}(\tau \rightarrow 3 e)}{\operatorname{Br}(\tau \rightarrow e \gamma)} \simeq \frac{1}{95}, \\
\frac{\operatorname{Br}(\tau \rightarrow e 2 \mu)}{\operatorname{Br}(\tau \rightarrow e \gamma)} \simeq \frac{1}{430} .
\end{gathered}
$$

Thus, from the experimental bound on $\operatorname{Br}(\tau \rightarrow \mu / e \gamma)$, we find that $\operatorname{Br}(\tau \rightarrow \mu 2 e)$ and $\operatorname{Br}(\tau \rightarrow 3 e)$ can reach $10^{-8}$, and $\operatorname{Br}(\tau \rightarrow e 2 \mu)$ and $\operatorname{Br}(\tau \rightarrow 3 \mu)$ can reach $10^{-9}$.

\section{B. EDMs of charged leptons}

The EDMs of charged leptons are induced essentially through the threshold correction to $A_{e}$ at the heavy singletneutrino scale, and the dependence on $H$ and $\mathcal{M}_{\nu}$ is compli- cated. From Eq. (22) it is found that the imaginary parts of diagonal terms in $A_{e}$ can reach $\mathcal{O}(0.1) \%$ if the $\left(X_{k}\right)_{i j}$ are no larger than $4 \pi$. In this case, the muon and electron EDMs can roughly reach the level of $10^{-25} e \mathrm{~cm}$ and $10^{-27} e \mathrm{~cm}$, respectively. However, the non-negligible contribution of the heavy singlet neutrino threshold correction may lead to large $\operatorname{Br}(\mu \rightarrow e \gamma)$ even if $H_{12}$ and $H_{13} H_{32}$ are suppressed. Therefore careful numerical study is required for the predictions of EDMs in the supersymmetric seesaw model.

We show first in Fig. 4 predictions for the EDMs of the muon and electron from a random sampling of the parameter space, as a scatter plot of the muon EDM against the leftsmuon mass for the ansatz $H_{1}$. As before, we take the $\mathrm{SU}(2)$ gaugino mass to be $200 \mathrm{GeV}, A_{0}=-3 m_{0}$ in order to maximize the EDMs, $\mu>0$, and $\tan \beta=10$. The parameters in $\mathcal{M}_{\nu}$ and $H$ are the same as in Fig. 1. We assume the normal hierarchy for the light neutrino mass spectrum, and impose the constraints from $\operatorname{Br}(\mu \rightarrow e \gamma)$ and $\operatorname{Br}(\tau \rightarrow \mu(e) \gamma)$. We find that the muon EDM can reach $10^{-(27-28)} e \mathrm{~cm}$ in this 

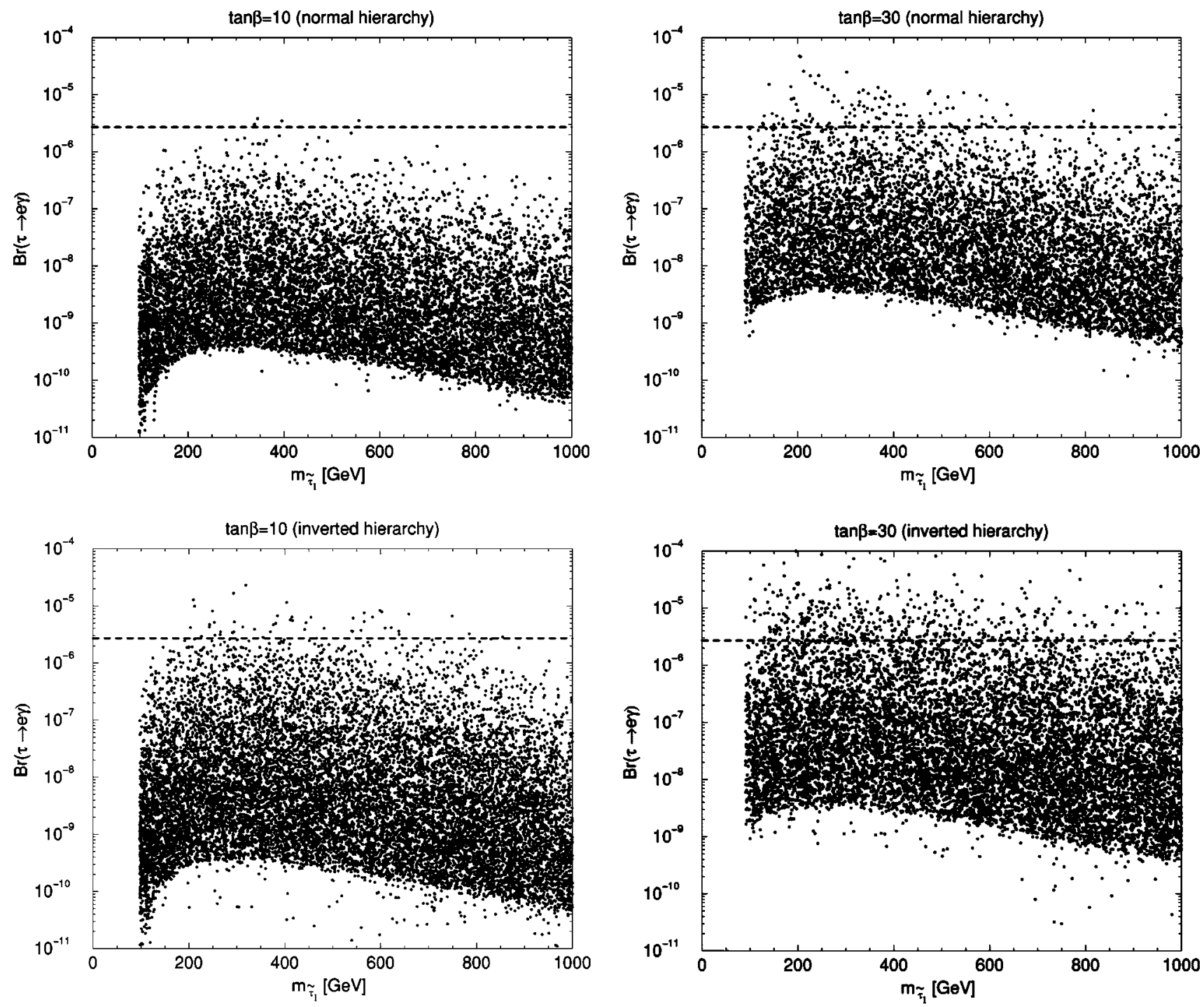

FIG. 3. Scatter plot of $\operatorname{Br}(\tau \rightarrow e \gamma)$ against the lightest stau mass for the ansatz $H_{2}$. The input parameters for the supersymmetry-breaking parameters are the same as in Fig. 2.
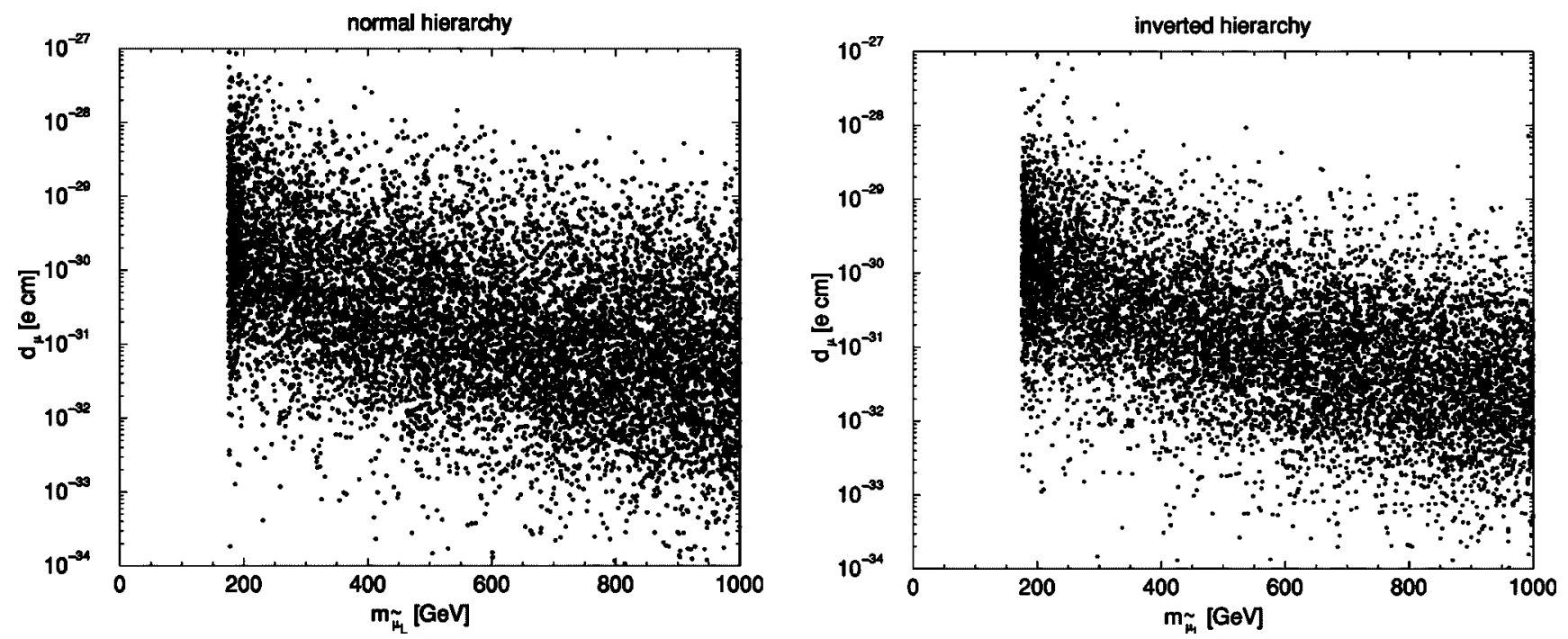

FIG. 4. Scatter plot of the muon EDM against the left-smuon mass for the ansatz $H_{1}$, taking the SU(2) gaugino mass to be $200 \mathrm{GeV}$, $A_{0}=-3 m_{0}, \mu>0$, and $\tan \beta=10$. We assume the normal hierarchy for the light neutrino mass spectrum in (a) and the inverted hierarchy in (b). 

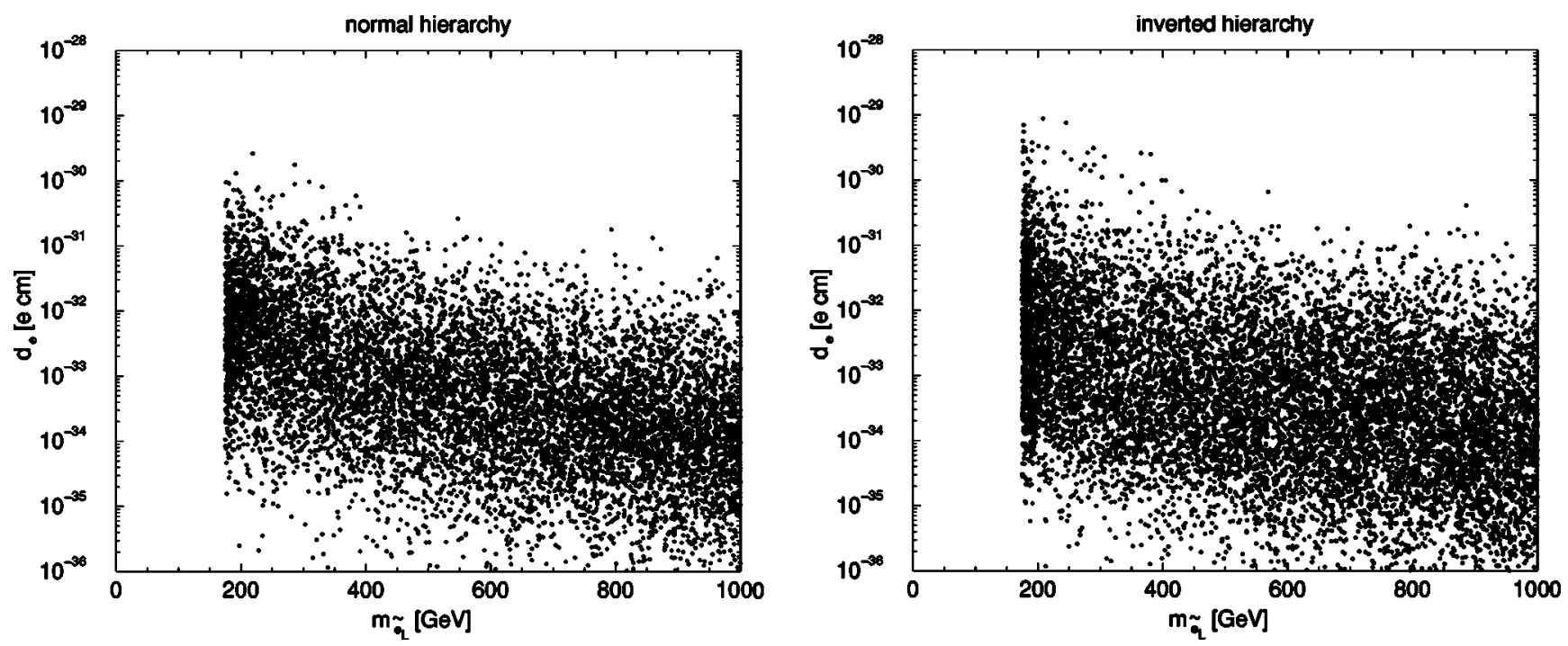

FIG. 5. Scatter plot of the electron EDM against the left-selectron mass for the ansatz $H_{2}$. Other input parameters are the same as in Fig. 4.

sampling, which is reasonable, since $\left(m_{\tilde{L}}^{2}\right)_{12}$ should suppressed by $10^{-(3-4)}$ because of the experimental bound on $\operatorname{Br}(\mu \rightarrow e \gamma)$. The EDM increases proportionally to $A_{0}$, as expected, but is insensitive to $\tan \beta$, if it is not extremely large.

Similarly, in Fig. 5 we show the electron EDM plotted against the left-selectron mass for the ansatz $\mathrm{H}_{2}$. We find that the electron EDM can reach $10^{-(29-30)} e \mathrm{~cm}$ in this sampling. Again, the main limiting factor is the experimental bound on $\operatorname{Br}(\mu \rightarrow e \gamma)$.

We recall that a proposal has been made to BNL that aims at a sensitivity of $10^{-24} e \mathrm{~cm}$ for the muon EDM [23], and the front end of a neutrino factory may be able to reach a sensitivity of $5 \times 10^{-26} e \mathrm{~cm}$ [21]. On the other hand, although the present upper limit on the electron EDM is 1.6 $\times 10^{-27} e \mathrm{~cm}$ [24], a technique has been proposed that may be sensitive to $10^{-32} e \mathrm{~cm}$ [25]. This would be sensitive to many of the models studied.

\section{CONCLUSIONS}

We have presented a new parametrization of the minimal seesaw mechanism, which enables the heavy neutrino Dirac Yukawa couplings and masses to be fixed in terms of the light neutrino parameters and a Hermitian parameter matrix $H$. In the minimal supersymmetric version of the seesaw model, the matrix $H$ can be related directly to low-energy physical observables. As a result, our parametrization is particularly suitable for comprehensive studies of the charged LFV processes and EDMs in supersymmetric models.

As applications, we have studied the LFV $\tau$ decays and the EDMs of the muon and electron in the minimal supersymmetric seesaw model. It is found that $\operatorname{Br}(\tau \rightarrow \mu(e) \gamma)$ could exceed the present experimental bounds, even when $\operatorname{Br}(\mu \rightarrow e \gamma)$ is suppressed much below the current limit. This implies that $B$ factories have a possibility of discovering LFV $\tau$ decays, since they may reach sensitivities $\operatorname{Br}(\tau$ $\rightarrow \mu(e) \gamma) \sim 10^{-8}$. The CERN Large Hadron Collider (LHC) may have a similar sensitivity, and a super $B$ factory may reach the level $10^{-9}$ for the same processes [22]. The LHC may also have a good sensitivity to $\operatorname{Br}\left(\tau \rightarrow \mu(e) \ell^{+} \ell^{-}\right)$. We show that $\operatorname{Br}(\tau \rightarrow \mu e e)$ and $\operatorname{Br}(\tau \longrightarrow 3 e)$ are about five times larger than $\operatorname{Br}(\tau \rightarrow 3 \mu)$ and $\operatorname{Br}(\tau \rightarrow e 2 \mu)$, due to the larger phase space, and can reach $\sim 10^{-8}$ and $\sim 10^{-9}$, respectively, from the experimental bounds on $\operatorname{Br}(\tau \rightarrow \mu(e) \gamma)$. Finally, in our random samples the EDMs of muon and electron can attain $10^{-(27-28)} e \mathrm{~cm}$ and $10^{-(29-30)} e \mathrm{~cm}$, respectively, while their perturbative bounds in this model are $\sim 10^{-25} e \mathrm{~cm}$ and $\sim 10^{-27} e \mathrm{~cm}$. The electron EDM, in particular, may be accessible to experiment [25].

We have restricted our discussion to the supersymmetric seesaw model in this paper. However, our framework is also suitable for studying supersymmetric GUT models with heavy singlet neutrinos. In these models, $K$ and $B$ physics are also interesting, because the right-handed squarks have flavor-violating masses, as a result of quark-lepton unification [26]. Since the right-handed sleptons have LFV masses, the relation between the LFV and our parametrization may be quite complicated [27]. However, if the prediction for the LFV masses for right-handed sleptons is used, our parametrization is applicable.

\section{ACKNOWLEDGMENTS}

We thank C. Peña-Garay and A. Strumia for discussions. This work is partially supported by EU TMR contract No. HPMF-CT-2000-00460, ESF grant No. 5135, the Grant-inAid for Scientific Research from the Ministry of Education, Science, Sports and Culture of Japan (No. 13135207 and No. 14046225), and the JSPS. 
[1] SNO Collaboration, Q.R. Ahmad et al., Phys. Rev. Lett. 89, 011301 (2002).

[2] SNO Collaboration, Q.R. Ahmad et al., Phys. Rev. Lett. 89, 011302 (2002); J.N. Bahcall, M.C. Gonzalez-Garcia, and C. Pena-Garay, J. High Energy Phys. 07, 054 (2002); V. Barger, D. Marfatia, K. Whisnant, and B.P. Wood, Phys. Lett. B 537, 179 (2002); P. Creminelli, G. Signorelli, and A. Strumia, J. High Energy Phys. 05, 052 (2001); A. Bandyopadhyay, S. Choubey, S. Goswami, and D.P. Roy, Phys. Lett. B 540, 14 (2002).

[3] J. Shirai, talk given in XXth International Conference on Neutrino Physics and Astrophysics, Munich, Germany, 2002.

[4] Super-Kamiokande Collaboration, Y. Fukuda et al., Phys. Rev. Lett. 81, 1562 (1998).

[5] M. Gell-Mann, P. Ramond, and R. Slansky, in Supergravity, Proceedings of the Stony Brook Workshop, New York, 1979, edited by P. van Nieuwenhuizen and D. Freedman (NorthHolland, Amsterdam, 1979); T. Yanagida, in Proceedings of the Workshop on Unified Theories and Baryon Number in the Universe, Tsukuba, Japan, 1979, edited by A. Sawada and A. Sugamoto (KEK Report No. 79-18, Tsukuba, 1979); R. Mohapatra and G. Senjanovic, Phys. Rev. Lett. 44, 912 (1980).

[6] F. Borzumati and A. Masiero, Phys. Rev. Lett. 57, 961 (1986).

[7] J. Hisano, T. Moroi, K. Tobe, M. Yamaguchi, and T. Yanagida, Phys. Lett. B 357, 579 (1995); J. Hisano, T. Moroi, K. Tobe, and M. Yamaguchi, Phys. Rev. D 53, 2442 (1996).

[8] S. Davidson and A. Ibarra, J. High Energy Phys. 09, 013 (2001).

[9] J. Hisano and D. Nomura, Phys. Rev. D 59, 116005 (1999); J. Hisano, hep-ph/0204100.

[10] J.A. Casas and A. Ibarra, Nucl. Phys. B618, 171 (2001).

[11] W. Buchmüller, D. Delepine, and F. Vissani, Phys. Lett. B 459, 171 (1999); M.E. Gomez, G.K. Leontaris, S. Lola, and J.D. Vergados, Phys. Rev. D 59, 116009 (1999); J.R. Ellis, M.E. Gomez, G.K. Leontaris, S. Lola, and D.V. Nanopoulos, Eur. Phys. J. C 14, 319 (2000); W. Buchmüller, D. Delepine, and L.T. Handoko, Nucl. Phys. B576, 445 (2000); J.L. Feng, Y. Nir, and Y. Shadmi, Phys. Rev. D 61, 113005 (2000); T. Blazek and S.F. King, Phys. Lett. B 518, 109 (2001); D. Carvalho, J. Ellis, M. Gomez, and S. Lola, ibid. 515, 323 (2001); S. Lavignac, I. Masina, and C.A. Savoy, ibid. 520, 269 (2001); Nucl. Phys. B633, 139 (2002); F. Deppisch, H. Pas, A. Redelbach, R. Ruckl, and Y. Shimizu, hep-ph/0206122.

[12] J. Sato and K. Tobe, Phys. Rev. D 63, 116010 (2001); J. Hisano and K. Tobe, Phys. Lett. B 510, 197 (2001).

[13] J.R. Ellis, J. Hisano, M. Raidal, and Y. Shimizu, Phys. Lett. B 528, 86 (2002).
[14] CHOOZ Collaboration, M. Apollonio et al., Phys. Lett. B 420, 397 (1998).

[15] F. Boehm et al., Phys. Rev. D 64, 112001 (2001).

[16] N. Arkani-Hamed, J.L. Feng, L.J. Hall, and H. Cheng, Nucl. Phys. B505, 3 (1997).

[17] N.V. Krasnikov, Phys. Lett. B 388, 783 (1996); N. ArkaniHamed, H.C. Cheng, J.L. Feng, and L.J. Hall, Phys. Rev. Lett. 77, 1937 (1996); M. Hirouchi and M. Tanaka, Phys. Rev. D 58, 032004 (1998); D. Nomura, ibid. 64, 075001 (2001); J. Hisano, M.M. Nojiri, Y. Shimizu, and M. Tanaka, ibid. 60, 055008 (1999); J. Hisano, hep-ph/9906312; K. Agashe and M. Graesser, Phys. Rev. D 61, 075008 (2000); I. Hinchliffe and F.E. Paige, ibid. 63, 115006 (2001); J. Hisano, R. Kitano, and M.M. Nojiri, hep-ph/0202129.

[18] J.R. Ellis, J. Hisano, S. Lola, and M. Raidal, Nucl. Phys. B621, 208 (2002).

[19] L. Barkov et al., "Search for $\mu^{+} \rightarrow e^{+} \gamma$ down to $10^{-14}$ branching ratio," available at http://www.icepp.s.u-tokyo.ac.jp/ meg

[20] MECO Collaboration, BNL Proposal AGS P940 1997; M. Furusaka et al., JAERI/KEK Joint Project Proposal, "The Joint Project for High-Intensity Proton Accelerators," KEKREPORT-99-4, JAERI-TECH-99-056.

[21] See, for example, J. Äystö et al., "Physics with Low-Energy Muons at a Neutrino Factory Complex," hep-ph/0109217.

[22] T. Ohshima, Neutrino Oscillations and Their Origin (NOON2001), talk at the workshop (ICRR, Univ. of Tokyo, Kashiwa, Japan, 2001).

[23] R. Carey et al., Letter of Intent to BNL (2000); Y.K. Semertzidis et al., hep-ph/0012087.

[24] B.C. Regan, E.D. Commins, C.J. Schmidt, and D. DeMille, Phys. Rev. Lett. 88, 071805 (2002).

[25] S.K. Lamoreaux, Phys. Rev. A 66, 022109 (2002).

[26] R. Barbieri, L.J. Hall, and A. Strumia, Nucl. Phys. B449, 437 (1995); T. Moroi, J. High Energy Phys. 03, 019 (2000); G. Barenboim, K. Huitu, and M. Raidal, Phys. Rev. D 63, 055006 (2001); S.W. Baek, T. Goto, Y. Okada, and K.I. Okumura, ibid. 63, 051701(R) (2001); 64, 095001 (2001); N. Akama, Y. Kiyo, S. Komine, and T. Moroi, ibid. 64, 095012 (2001); M.B. Causse and J. Orloff, Eur. Phys. J. C 23, 749 (2002); D. Chang, A. Masiero, and H. Murayama, hep-ph/0205111.

[27] R. Barbieri and L. Hall, Phys. Lett. B 338, 212 (1994); R. Barbieri, L. Hall, and A. Strumia, Nucl. Phys. B445, 219 (1995); B449, 437 (1995); P. Ciafaloni, A. Romanino, and A. Strumia, ibid. B458, 3 (1996); J. Hisano, T. Moroi, K. Tobe, and M. Yamaguchi, Phys. Lett. B 391, 341 (1997); 397, 357(E) (1997); J. Hisano, D. Nomura, Y. Okada, Y. Shimizu, and M. Tanaka, Phys. Rev. D 58, 116010 (1998); J. Hisano, D. Nomura, and T. Yanagida, Phys. Lett. B 437, 351 (1998); S.F. King and M. Oliveira, Phys. Rev. D 60, 035003 (1999). 